\title{
Comparative study of various mechanisms for metallocene-catalyzed $\alpha$-olefin polymerization
}

\author{
Iñaki Silanes and Jesus M. Ugalde
}

Kimika Fakultatea, Euskal Herriko Unibertsitatea and Donostia International Physics Center (DIPC), P.K. 1072, 20080 Donostia, Euskadi, Spain

\section{References}

[13] Das, K.; Doskter, D. W.; Fahey, D. R.; Lauffer, D. R.; Hawkins, G. D.; Li, J.; Zhu, T.; Cramer, C. J.; Truhlar, D. G.; Dapprich, S.; Froese, R. D. J.; Hothausen, M. C.; Liu, Z.; Mogi, K.; Vyboishchikov, S.; Musaev, D. G.; Morokuma, K. in Transition State Modeling for Catalysis, Truhlar, D. G.; Morokuma, K., Eds. volume ACS SympSer 721. 1998.

[39] Frisch, M. J.; Trucks, G. W.; Schlegel, H. B.; Scuseria, G. E.; Robb, M. A.; Cheeseman, J. R.; Zakrzewski, V. G.; Montgomery, J. A.; Stratmann, R. E.; Burant, J. C.; Dapprich, S.; Millam, J. M.; Daniels, A. D.; Kudin, K. N.; Strain, M. C.; Farkas, O.; Tomasi, J.; Barone, V.; Cossi, M.; Cammi, R.; Mennucci, B.; Pomelli, C.; Adamo, C.; Clifford, S.; Ochterski, J.; Petersson, G. A.; Ayala, P. Y.; Cui, Q.; Morokuma, K.; Malick, D. K.; Rabuck, A. D.; Raghavachari, K.; Foresman, J. B.; Cioslowski, J.; Ortiz, J. V.; Stefanov, B. B.; Liu, G.; Liashenko, A.; Piskorz, P.; Komaromi, I.; Gomperts, R.; Martin, R. L.; Fox, D. J.; Keith, T.; Al-Laham, M. A.; Peng, C. Y.; Nanayakkara, A.; Gonzalez, C.; Challacombe, M.; Gill, P. M. W.; Johnson, B. G.; Chen, W.; Wong, M. W.; Andres, J. L.; Head-Gordon, M.; Replogle, E. S.; Pople, J. A. Gaussian 98, a.11.3. Gaussian, Inc., Pittsburgh PA, 2002. 